\title{
Adaptive Coexistence of Delay Sensitive and Delay Tolerant Devices: A Control Theoretic Approach
}

This paper was downloaded from TechRxiv (https://www.techrxiv.org).

\section{LICENSE}

CC BY 4.0

SUBMISSION DATE / POSTED DATE

08-02-2022 / 10-02-2022

\section{CITATION}

Pokhrel, Shiva Raj; Choi, Jinho (2022): Adaptive Coexistence of Delay Sensitive and Delay Tolerant Devices: A Control Theoretic Approach. TechRxiv. Preprint. https://doi.org/10.36227/techrxiv.19136348.v1

$\mathrm{DOI}$ 


\title{
Adaptive Coexistence of Delay Sensitive and Delay Tolerant Devices: A Control Theoretic Approach
}

\author{
Shiva Raj Pokhrel, senior member, IEEE and Jinho Choi, senior member, IEEE
}

\begin{abstract}
We consider future cellular networks and study the coexistence of delay-sensitive (DS) and delay-tolerant (DT) devices in machine-type communication (MTC). DS devices require to minimize access delay for their low delay requirements; in contrast, DT devices have flexible delay constraints. For reducing access delay, we extend our fast retrial idea [1] in the data transmissions when a group of preambles is divided into two subsets to support the time-varying nature of the traffic from DS devices. We focus on the stability dynamics by design-we derive convergence conditions by using a control-theoretic approach in terms of variation in (i) the arrival rates, (ii) buffer sizing at the devices, (iii) the number of preambles, and (iv) the number of DS devices. More importantly, we develop a novel adaptive algorithm that dynamically allocates the number of preambles for DS, thus guaranteeing stability by design. We validate our findings with extensive simulations. Besides, we develop a novel framework that describes how the control theory idea can be applied to address the issue of how to track the buffers and handle coexistence in such a diverse network environment under realistic application constraints. Our extension to the control-theoretic idea predicts whether the overall system is stable, i.e. whether data flows are desynchronized. Given the data flows are desynchronized, small buffers are always sufficient. Our approach shows that a smaller DS buffer often promotes desynchronization-a virtuous cycle.
\end{abstract}

Index Terms-MTC; Random Access; Delay Tolerant; Delay Sensitive; Fast Retrial; Stability Analysis

\section{INTRODUCTION}

Smart cities primarily rely on the Internet-of-Things (IoT) to function. Fifth-generation $(5 \mathrm{G})$ cellular systems are enabling technology for the IoT over a large geographical area (e.g., metropolitan areas or regions). Therefore, $5 \mathrm{G}$ plays a crucial role in smart cities and any other IoT applications [2]-[4]. In addition, 5G will execute a vital role in allowing information gathered through sensors to be transmitted in real-time to convenient monitoring stations. This network of connectivity will facilitate continuance of the communication systems, as well as robust data flow control, dynamic adjustment of functioning parameters to react timely to the real-time variations in their network scenario and settings, as and when they occur [5], [6].

Machine type communication (MTC) plays a crucial role in promoting several IoT use cases within 5G systems [5]. Random Access Channel (RACH) [6], in particular, slotted ALOHA (Additive Links On-line Hawaii Area) has been widely investigated for MTC [7], as it is expected to maintain a large volume of MTC devices with sporadic data traffic.

S. R. Pokhrel and J. Choi are with the School of Information Technology, Deakin University, Geelong, VIC 3220, Australia (e-mail: shiva.pokhrel@deakin.edu.au,jinho.choi@deakin.edu.au).

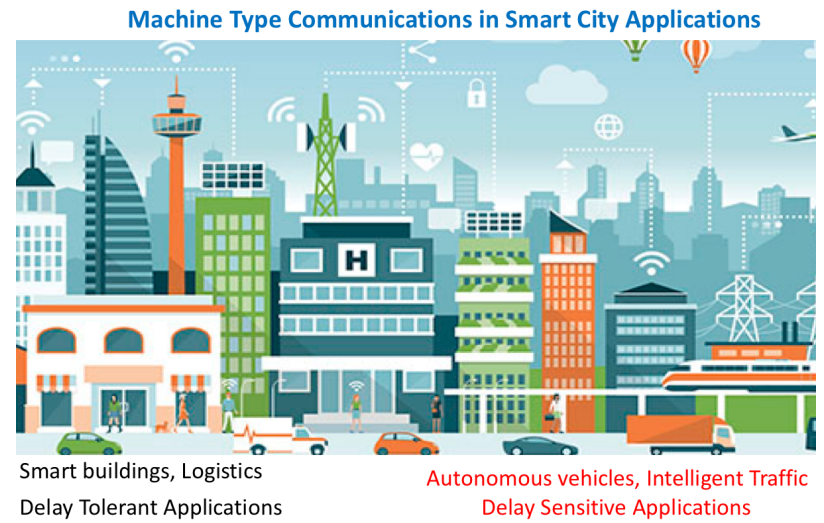

Fig. 1. Motivating scenario: A schematic view of 5G machine type communication in smart cities. 5G support massive machine type communication (mMTC) and critical machine type communication (cMTC).

In MTC RACH, a device is said to be an active device if it is holding at least one packet to be transmitted. An active device uses a preamble from a pool of preambles, which is shared by the contending devices, in multiple access timeslotted manners. Given a finite number of preambles in the pool, collision due to contention in such multiple access is inevitable, which occurs when two or more devices select and transmit the same preamble. Therefore the overall performance depends on the size of the preamble pool. For a given number of devices, it is worth noting that the frequency of collisions increases with the decrease in the size of the preamble pool. Thus, as reported in [8], it is essential to consider adjusting the volume of the preambles adaptively pool depending on the time-varying activity of the devices. Furthermore, an access class barring (ACB) scheme has been proposed for addressing the access control when the pool of preambles in MTC is of fixed size [9].

In general, 5G advises two types of MTC, namely massive machine-type and critical machine-type communications. Massive MTC is proposed for a vast number of IoT things, definitely a large number of sensors and actuators transferring many data back and forth. A motivating example of a smart city scenario is shown in Fig. 1. Use cases include smart buildings, logistics and fleet management, as well as air and water quality monitoring. It is designed to be delay-tolerant (DT), suited for small data blocks to be transmitted or received, and to be sent on low bandwidth channels.

Critical MTC is aimed for purposes where the data is delay-intolerant, so-called delay-sensitive (DS), and the crucial nature of such data requires assured, timely and precise 
TABLE I

LITERATURE ON PREAMBLE ALLOCATION FOR CONGESTION AVOIDANCE OF CELLULAR RACH

\begin{tabular}{|c|c|c|}
\hline References & RACH Scheme & Basic Concepts \\
\hline [5], [10], [11] & Back-off based & $\begin{array}{l}\text { When collisions occur, devices retransmit after an MTC- } \\
\text { specific duration of back-off }\end{array}$ \\
\hline$[11],[12]$ & $\mathrm{ACB}$ & $\begin{array}{l}\text { It assigns different access probabilities to multiple access } \\
\text { levels of devices. }\end{array}$ \\
\hline [13] & Extended ACB & $\begin{array}{l}\text { Specific class of devices accessing from the channel } \\
\text { control is prohibited }\end{array}$ \\
\hline$[14],[15]$ & Dynamic ACB & $\begin{array}{l}\text { Parameters are dynamically updated based on preceding } \\
\text { collisions. }\end{array}$ \\
\hline [16] & Collaborative ACB & $\begin{array}{l}\text { Parameters are designed by multiple stations in a coop- } \\
\text { erative fashion. }\end{array}$ \\
\hline [17], [18] & Prioritized dynamic ACB & Uses back-offs dependent on class, and adaptive ACB. \\
\hline$[5]$ & Slotted RACH & $\begin{array}{l}\text { Dedicated random access slot for each device, and is } \\
\text { allowed to transmit only in that slot. }\end{array}$ \\
\hline [5], [19]-[21] & Separated RACH & $\begin{array}{l}\text { Available preambles or slots are divided between two } \\
\text { types of devices }\end{array}$ \\
\hline$[1],[22],[23]$ & Fast retrial & $\begin{array}{l}\text { Devices can immediately re-transmit after collisions } \\
\text { where preambles or slots are separated }\end{array}$ \\
\hline [5], [19] & Dynamic congestion avoidance & $\begin{array}{l}\text { Congestion is modelled at the station, and additional } \\
\text { RACH resources are dynamically assigned. }\end{array}$ \\
\hline [5] & Pull/Paging-based RACH & $\begin{array}{l}\text { Devices only make transmission attempts after they have } \\
\text { received paging from the station. }\end{array}$ \\
\hline This paper & Dynamic separated Fast retrial & $\begin{array}{l}\text { Dynamic separated RACH with Fast retrial for a time- } \\
\text { varying rate of data flows and devices }\end{array}$ \\
\hline
\end{tabular}

transmission to the destination [24].

Examples of critical MTC include unmanned purposes such as traffic safety, autonomous vehicles and electric grid control. As we can see, both of these DS and DT services are very much aligned with the vision of digitally automating our cities. In this paper, we consider co-existing DS and DT devices in an MTC system with a shared pool of preambles [1], [15]. Two important ideas for the future MTC system are as follows:

- If an IoT device can operate in a dual-mode, both as DS or DT device, based on the associated applications, it is essential to support both types of devices in a single dynamical system;

- If the number of DS devices is time-varying in a critical MTC sub-system, the resource for the massive MTC subsystem has to be adaptively allocated, which may result in complicated resource allocation for an entire dynamical system.

To address such advancements in 5G, an extensive MTC framework (that supports both DS and DT devices) can be efficient as the resource can be dynamically allocated within the system. Overall, our objective is the development of techniques for the analysis and design of the next generation of efficient, robust preamble allocation algorithms for an MTC system with co-existing DS and DT devices.

\section{A. Related Works and New Ideas}

To fix the issue of network congestion in cellular systems, 3GPP has defined several resource allocation schemes [25]: (i) ACB; (ii) back-off based; (iii) dynamic congestion avoidance; (iv) slotted RACH; (v) separated RACH; (vi) pull-based $\mathrm{RACH}$, etc. We have enlisted existing schemes in Table I, along with their fundamental concepts and the accompanying references.

Of particular relevance to this work are the variants of separated RACH [5], [19], [20], [22], [23] and dynamic ACB schemes [14], [15]. However, none of these works [5], [14], [15], [19]-[23] considered the scalable tuning of preamble pool for DS devices and their dynamic allocation without prior knowledge of data flow rates from the DS devices.

The RACH contention mechanism has been extensively investigated not only to distribute a load of access traffic over multiple time slots in order to prevent arbitrary access behaviors, but also to tune system settings depending on MTC access loads. While most ACB schemes are successful only for DT devices, it is plausible to extend so that DS devices can be taken into account for preamble allocation to the two groups. For example, Du et al. [21] employed Newton's iterative approach and studied dynamic allocation of preambles.

With relevant insights from our earlier works [1], [4], [23] and others [21], we develop a dynamic separated fast retrial approach for tackling the time-evolving traffic intensity of DS devices and their temporal variation. Note that the approach in [21] is considered for four-step RACH, while our approach is for two-step RACH.

Although there are various retransmission techniques for the MTC system, a fast retransmission [22] should be one of the tempting and beneficial schemes for maintaining its low delay requirements, where another randomly picked preamble is to be adopted instantly in the following slot. In [20], [26], [27], a thorough performance study of 4-step random access methods was considered, and separate entity retransmission strategies and their delay aspects have been analyzed in detail. As mentioned, we have recently analyzed the delay performance of the 2-step random access, with fast retrial [1], [4]. The analysis in [1], [4], [23] of multichannel ALOHA using the notion of effective bandwidth and capacity help us to see delay output in terms of the quality-of-service and queue length. However, the stability analysis of the overall MTC system dynamics remains poorly understood [1], [23].

Nevertheless, in contrast to the aforementioned works (recall 
Tab. I), we deal with large multiplexers, which implies we let the packet service rate be equal to the data flows and consider a situation when it is very high. Observe that we can pick the buffer size in many ways. We require buffer size to be proportional to the data flows in the large buffer system, which is the basic thumb rule-selecting buffer size equivalent to bandwidth delay. However, with a large buffer, the queuing delay will then be relatively high, so large buffering are only suited only for the DT devices (but not desirable for DS).

For DS devices, different from [1], [4], [23], the queuing delay can be minimized with smallish fixed buffer size and statistical multiplexing. It is worth noting that various methods from queueing theory can be utilized to demonstrate that both of these schemes lead to different models of fluids. The basis of our novel approach is an account of the relative timescales of the queueing events tightly coupled with the aspects of data flow control.

We are inspired by the success of the queuing process in [1], [4]. It was also used to answer a somewhat similar problem in [28], from a higher layer perspective, though using different theoretical techniques under different network settings. We investigate the stability dynamics of the entire MTC system with a dynamic separated fast retrial approach and their data flow control by considering different buffer size regimes for the DS and DT devices.

\section{B. Our Contributions}

Our main contributions in this paper are as follows:

$\mathbf{C}_{1}$. We develop a robust framework for designing a low delay-based adaptive preamble allocation algorithm based on our fast retrial idea [1]. When the pool of preambles is divided into DS/DT subsets, our new framework applies a controltheoretic approach to support the time-varying nature of the traffic from DS devices and their temporal fluctuations.

$\mathbf{C}_{2}$. We derive conditions to guarantee the stability of data flow dynamics by design - we formulate stability constraints by using a control-theoretic approach in terms of variation in (i) the arrival rates; (ii) buffer sizing at DS devices; (iii) the number of preambles; and (iv) the number of DS devices.

$\mathbf{C}_{3}$. More importantly, we develop a novel adaptive algorithm that dynamically allocates the number of preambles for DS devices, thus guaranteeing stability by design. Our findings have been validated with extensive simulations.

It is worth noting that, compared to earlier works [1], [4], [23], the approach in the paper can be viewed as a comprehensive framework based on a control-theoretic idea, which provides more options to design adaptive preamble allocation in time-evolving MTC systems with co-existing DS and DT devices.

\section{Network Scenario AND SETTING}

We consider a two-step RACH approach for a system consisting of a base station (BS) and both DS and DT types of MTC devices. We have the following assumption.

ASSUMPTION 1. DS devices have low-latency requirements while DT devices are always saturated and backlogged with packets but do not have any delay requirements.

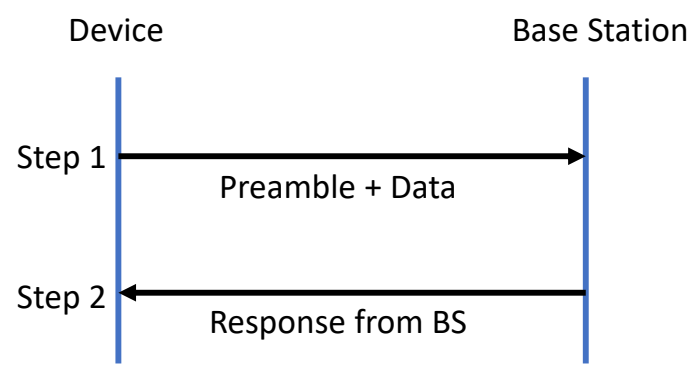

Fig. 2. Two-step random access mechanism in MTC.

As suggested in [1], [4], [23], [29], Assumption 1 is an important and justified fact, i.e., preambles can be reserved for DS devices. Fig. 3 shows the division of contention-based preambles between DS and DT devices. Priority can be set via preamble reservation for DS devices when the total number of reserved preambles is given. Reserving preambles for the coexisting DS/DT MTC scenario can reduce the collision probability of the DS devices. However, as DS devices transmit sporadically and unused reserved preambles often waste the bandwidth and increase the collision probability of the DT devices. Therefore, as argued in [29], in the coexisting DS/DT MTC system, Assumption 1 is motivated with an underlying justification that the number of DS devices is generally much smaller than that of DT devices, while DS devices still demand more resources to meet their low delay requirements. Relaxation of this assumption with relevant insights from this work, i.e., a special case where DT devices are delay-constrained requires further investigations and will be studied in future.

\section{A. Two-Step Random Access}

Fig. 2 shows how two-step approach works. The first and third steps in 4-step random access [25] are to transmit a randomly selected preamble and a data packet, respectively, while these two steps are combined into the first step in the two-step random access. In the second step, the BS is to send a feedback signal so that devices can see whether or not collisions happen. In particular, the BS sends the identity sequence within if a packet is successfully decoded. Then, a device can compare the identity sequence in the message with the identity sequence transmitted by the BS in the second step. If they are the same, the device can see that its packet is successfully transmitted. Otherwise, the device assumes that its packet is not successfully decoded. In this case, it can drop messages or try to re-transmit according to re-transmission strategies.

The initial step of the two-step random access has two phases: the preamble phase and the data transmission phase. In the first preamble phase, a randomly selected preamble is transmitted, which is then followed by a data packet transmission in the second phase. See details in Fig 3. An active device transmits the data packet in the data transmission phase.

With two-step random access, our second assumption is as follows. 
AsSUMPTION 2. The length of the packet is less than or equal to that of the data transmission phase, and a preamble collision is equivalent to a packet collision.

ASSUMPTION 2 is to guarantee that a packet fits completely into a single slot as it is always valid for short message transmissions. In MTC protocols including two-step random access, a set of preambles is used to identify the devices that transmit signals. In general, the BS has a bank of correlators (each correlator is to detect a preamble). The output of a correlator is used to detect its corresponding preamble (e.g., the $l$ th correlator is to detect the $l$ th preamble) transmitted through the physical random access channel (PRACH). There can be multiple devices that choose the same preamble. In this case, since those devices transmit their data packets through the corresponding resource block in the physical uplink shared channel (PUSCH), they experience packet collisions. Therefore, we assume that a preamble collision (i.e., the case that multiple devices choose the same preamble) is equivalent to a packet collision. This approach is compatible with the standard in $5 \mathrm{G}$ [30], [31].

\section{B. Random Access for DS and DT Devices}

In mission-critical applications, since DS devices would need to send their messages within a limited time, it seems that contention-based transmissions (leading to collisions and retransmissions) will not be desirable. Thus, dedicated preambles could be one promising approach [32], which however limits the number of DS devices regardless of their activity. Another reliable approach to support more DS devices with lower access delay is the fast retrial [1], [22], [23] for contention-based transmissions.

As proposed in [1], [15], for a heterogeneous MTC system where DS and DT devices co-exist, a pool of preambles can be dynamically divided so that a sufficient number of preambles can be allocated to DS devices for a short access delay. In this case, since the remaining preambles are assigned to DT devices, they will compete separately for in a small number of preambles, which results in low throughput. Therefore, it is important to understand and quantify this trade-off for an adaptive and optimal preamble design in such a time-evolving heterogeneous MTC system of DS and DT devices.

\section{Preamble Allocation}

For two-step random access, we consider orthogonal $L$ preambles. $L_{1}(\leq L)$ preambles are assigned to DS devices, while $L_{2}=L-L_{1}$ preambles are assigned to DT devices. In the first step of the two-step RACH approach, each active device transmits a packet in a slot, where a preamble is randomly selected (an active DS device can randomly choose one

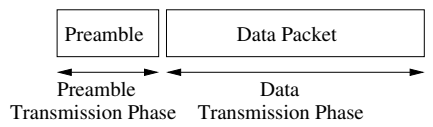

Fig. 3. Two phases (i.e., preamble transmission and data transmission phase) for the first step in 2-step random access. out of $L_{1}$ preambles, while an active DT device can randomly choose one out of $L-L_{1}$ preambles). For convenience, let

$$
\begin{aligned}
& \mathcal{L}_{1}=\left\{1, \ldots, L_{1}\right\} \\
& \mathcal{L}_{2}=\left\{L_{1}+1, \ldots, L\right\},
\end{aligned}
$$

where $\mathcal{L}_{1}$ and $\mathcal{L}_{2}$ denote the pools of preambles for DS and DT devices, respectively.

\section{Fast Retrial for DS Devices}

For minimizing access delay, any active DS device experiencing preamble collision can immediately re-transmit another randomly selected preamble in $\mathcal{L}_{1}$ without back-off using fast retrial approach [22].

In Fig. 4, we illustrate an example of a fast retrial approach with $\mathcal{L}_{1}=4$ preambles. At slot $t$, suppose that devices 1 and 3 transmit preamble 1, which results in preamble collision. At slot $t+1$, the two devices can re-transmit randomly selected preambles (preamble 2 for device 1 and preamble 4 for device 3 ), while any other active device, such as device 2 in Fig. 4 (which was inactive in the earlier slot), transmits preamble 1. Observe that all the devices successfully transmit their preambles. This simple example shows that immediate retransmission using a fast retrial approach may not always lead to successive collisions, however, reduces the access delay considerably (wastage due to back-off time).
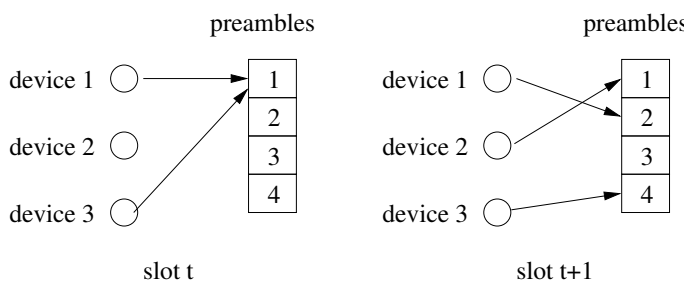

slot $\mathrm{t}+1$

Fig. 4. An example of a fast retrial approach consisting of three DS devices and four preambles.

\section{Adaptive Preamble Allocation Theory and DESIGN}

In this section, we describe our framework and the underlying design approach based on the three following different complex modules: (i) the adaptive preamble allocation; (ii) queuing analysis; and (iii) guaranteed stability by design. The design and analysis in this paper is based on the following successful tools: (a) fluid flow approach (being an extensively used approach in the design of network utility maximization [33]); (b) a stochastic queuing model (to capture the Spatio-temporal queue occupancy); (c) control-theoretic delay differential scheme (to quantify the time-varying dynamics in the channel); and (d) a Hopf-bifurcation analysis (for analyzing the stability of the different components during algorithm design).

We briefly explain the aforementioned tools as follows.

a) Fluid-flow method aggregates out the small stochastic variations in quantities of interest such as queue size, and typically produces ordinary differential equations to capture and explain 
the system dynamics. It substantially simplifies the process of the system analysis while maintaining the pragmatic facets of its behaviour. Many fluid models can therefore be based on a straightforward calculus approximation and fundamental principle of probability. In the approximation of data transport by a fluid-flow method [33], the number of data packets that are transmitted during a cycle duration of $T$ seconds long when the transmitting window ${ }^{1}$ is of $w(t)$ packets and round trip delay of $\tau(t)$ can be estimated as [33]

$$
\int_{0}^{T} \frac{w(t)}{\tau(t)} \mathrm{d} t
$$

where a cycle is the period during which $w(t)$ varies between the minimum and maximum size). The underlying approximation is that the variation of $w(t)$ is linear within the cycle duration, and always reaches its maximum. We adopt a similar notion for the preamble allocation in this work.

b) From the queuing theory perspective, arriving jobs get into the queues; in our setting the arriving jobs are packets that enter either the DS buffer or the DT buffer. These packets arriving into the queue, possibly wait some time for the preceding packets to be served, take some time being processed, and then depart from the queue, which leads to a queuing delay. More specifically, queuing delay is the time a packet waits in a queue until it can be transmitted, which is a key component of a network delay. Queue occupancy reflects the number of packets on the buffer, which is defined by the ratio of occupant length of packets to buffer size. As packet size and service times are generally constant, the queuing delay can be captured by tracking the time-varying occupancy of the queues.

c) The initial step for the control-theoretic analysis is to put down a series of nonlinear differential equations for all the rates of all the data flows in the network. For the likelihood of time-varying data rates in the devices of the system, we need to decide if such a complex system is stable or not.

The Lax equivalence theorem in the numerical analysis of finite difference methods is fundamental for solving the differential equations. It states that for a consistent finite difference method and well-posed linear initial value problem, the method is convergent if and only if it is stable. See Dahlquist's equivalence theorem (stability + consistency $\Longleftrightarrow$ convergence) for details.

Stability, in this context, is literally the control-theoretic concept used to check the desynchronization of the data flows (see Remark 1 for details). The principle we present here is aimed at determining whether synchronization occurs, and to what degree. A generalized nonlinear model for the resource allocation of data transport with multiple flows [28] is

$$
\frac{d w(t)}{d t}=(i(w(t))-d(w(t) b(t-\tau))) \frac{w(t-\tau)}{\tau},
$$

where $i(w(t))$ and $d(w(t))$ are the current increase and decrease in $w(t)$, respectively, and $b(t)$ is the likelihood of buffer being full.

\footnotetext{
${ }^{1}$ The transmitting window is a state variable that limits the volume of data a device can send into network (see [33] fro details.
}

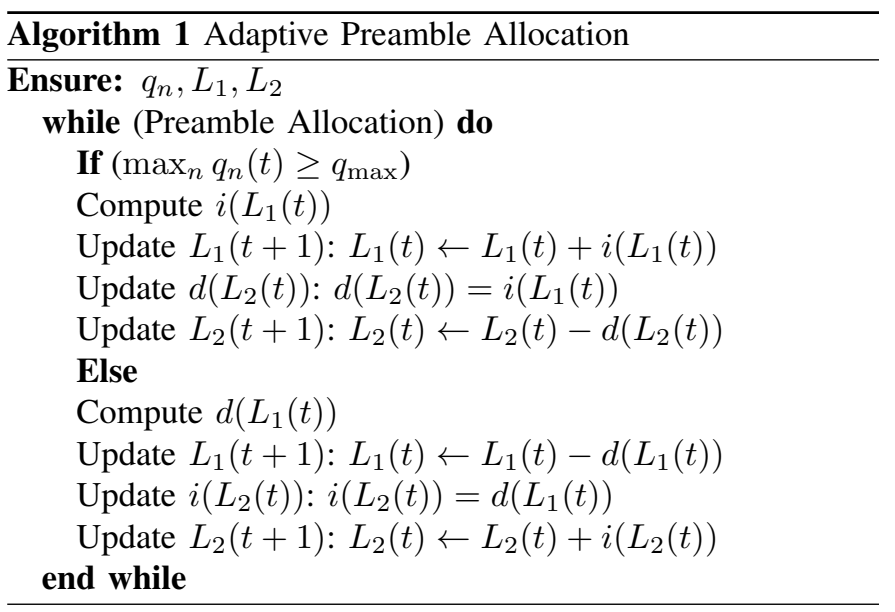

Observe that (2) provides us with another way to look for limit theorems which claim that in some idealized system, a given fluid model is obtained asymptotically, for instance, as the number of DS flows increases. Limit theorems will alert us to effects that are difficult to detect with simulations - we can identify several instabilities that we expect are only seen in systems of more than 4,000 devices or so flows, typically hard-to-simulate with standard software tools.

d) Using Hopf bifurcation, for a non-linear differential equation with a single discrete delay, we can conduct the calculations required to evaluate its stability and study its asymptotic aspects of the bifurcation for the solutions. For resource allocation algorithms based on such solutions, we can analyze the lack of local stability and assess the adaptive dual control algorithms [28]. Besides, we could quantify the influence of the device/network parameters on the oscillations and bifurcations (if any) [34]. We have adopted a few control insights from adaptive dual control algorithms [28], [34] for designing implementable adaptive preamble allocation (see Sec. III-E ).

\section{A. Adaptive Preamble Allocation}

Let $N_{1}$ be the total number of DS devices. For tractability similar to [1], [4], let $K_{1}(t)\left(\leq N_{1}\right)$ denote the number of active DS devices contending for sending preambles at slot $t$.

At the $n$th active DS device, the occupancy state of the queue satisfies the following recurrence relation:

$$
q_{n}(t+1)=\left(q_{n}(t)+a_{n}(t)-s_{n}(t)\right)^{+}, n=1, \ldots, N_{1},
$$

where $q_{n}(t), a_{n}(t)$, and $s_{n}(t)$ are the length of queue, the number of new arrivals (of access request), and the number of successful preamble transmissions of the $n$th DS device at slot $t$, respectively. Here, $(x)^{+}=\max \{0, x\}$. Note that a DS device becomes active if its queue is not empty, i.e., $q_{n}(t)>0$, with the fast retrial.

Let $L_{1}(t)$ and $L_{2}(t)$ denote the numbers of preambles allocated to DS and DT devices at slot $t$, respectively. The proposed adaptive preamble allocation scheme is shown in Algorithm 1 and explained as follows. Since each active device randomly chooses a preamble from the pool, it can be shown that

$$
s_{n}(t)= \begin{cases}1, & \text { w.p. } p_{n}(t) \\ 0, & \text { w.p. } 1-p_{n}(t)\end{cases}
$$


where $p_{n}(t)=\left(1-\frac{1}{L_{1}(t)}\right)^{K_{1}(t)-1}$ is the conditional probability of no preamble collision or successful preamble transmission when there are $K_{1}(t)$ active DS devices.

Remark 1 (Interrelationship of (3) and (4)): In (3), $q_{n}(t)$ stands for the number of packets in the queue (buffer) of device $n$. In two-step random access, since the length of packet is usually fixed, the number of packets is sufficient to express the length of queue (i.e., $q_{n}(t)$ can be seen as the normalized length of queue). For example, letting $D$ be the length of packets in bits, the actual queue length can be $D q_{n}(t)$, while $D$ is dropped as it is a fixed and known quantity. As a result, the number of new arrivals can be seen as the number of new access requests at each device. In twostep random access, each access is equivalent to one packet transmission as a packet transmission immediately follows a preamble transmission (Assumption 2).

As shown in Algorithm 1, if the queue length of the $n$th DS device is greater than or equal to $q_{\max }$, it can send a reserved preamble, which is referred to as preamble 0 , to inform the BS. Then, the BS can increase the number of preambles by $i\left(L_{1}(t)\right)$ for all DS devices, otherwise decrease by $d\left(L_{1}(t)\right)$ as follows:

$$
L_{1}(t+1)=L_{1}(t)+\Delta L_{1}(t),
$$

where

$$
\Delta L_{1}(t)= \begin{cases}i\left(L_{1}(t)\right), & \text { if } \max _{n} q_{n}(t) \geq q_{\max } \\ d\left(L_{1}(t)\right), & \text { o.w. }\end{cases}
$$

Note that multiple DS devices can send preamble 0 simultaneously. Since the same preamble is transmitted, although they have collided, the BS can detect the presence of preamble 0 and update $L_{1}(t)$ as shown in (5).

As indicated in Algorithm 1, the corresponding allocation for the DT preambles due to the adaptive allocation of DS preambles can be quantified as follows. The increase in the number of preambles by $i\left(L_{2}(t)\right)$ for DT devices or decrease by $d\left(L_{2}(t)\right)$ due to the corresponding decrease and increase in the $L_{1}(t)$ (as shown in (5)):

$$
L_{2}(t+1)=L_{2}(t)+\Delta L_{2}(t),
$$

where

$$
\Delta L_{2}(t)= \begin{cases}d\left(L_{2}(t)\right), & \text { if } \Delta L_{1}(t)=i\left(L_{1}(t)\right) \\ i\left(L_{2}(t)\right), & \text { o.w. }\end{cases}
$$

and

$$
i\left(L_{2}(t)\right)=d\left(L_{1}(t)\right) \text { and } d\left(L_{2}(t)\right)=i\left(L_{1}(t)\right) .
$$

\section{B. Mathematical Interpretation of the Queuing}

Our mathematical interpretation of the queuing process at a DS device is as follows. Let $\lambda_{n}$ be the rate at which access request arrives at device $n$, and $\mu_{n}$ be the successful transmission rate. The probability that the DS queue is fully occupied can be approximated by the blocking probability of an $M / D / 1$ queue with a small size buffer (low delay) $q_{\max }$, Poisson arrival rate $\lambda_{n}$ and service rate $\mu_{n}$.
We approximate the blocking probability, $b(t)$, to estimate the full queue occupancy of a small DS buffer $q_{n}(t)$ as

$$
\begin{aligned}
b(t) & =\operatorname{Pr}\left(\max _{n} q_{n}(t) \geq q_{\max }\right)=\left(\max _{n} \frac{\lambda_{n}(t)}{\mu_{n}(t)}\right)^{q_{\max }} \\
& \approx\left(\max _{n} \frac{a_{n}(t)}{p_{n}(t)}\right)^{q_{\max }},
\end{aligned}
$$

where we assume same observation time to compute the service and the arrival rate.

We can see from (9), that when arrivals $a_{n}(t)$ are higher, DS devices in our setting will perceive higher blocking $b(t)$ thus demanding more preambles from the BS to drain their queues and ameliorate queuing delays. This is the model of the adaptive allocation algorithm proposed in this paper. (9) has been useful for the stability analysis of the system dynamics (Sec. III-C).

Remark 2 (Desynchronization of data flows): Observe that for a DS device, when preamble increments (resp. decrements), the queue drains (resp. fills up), and then the link idles (resp. backlogged) as the queue underflows (resp. overflows). When several data flows from $N_{1}$ devices are all synchronized (i.e. there is a continuous oscillation between this idle and busy phenomenon, which worsens the system with several underflows and overflows, and the preamble allocation never stabilizes). This unstable phenomenon is undesirable, as the system dynamics neither operate optimally nor becomes stable. Therefore, for synchronized data flows from $N_{1}$ devices, the required preamble allocation is very high i.e., $N_{1} L_{1}$. In sharp contrast to this, when data flows are fully desynchronized, there are no such oscillations. Furthermore, by applying the central limit theorem, we can see that the aggregate preamble allocation required for the $N_{1}$ DS devices follows Normal distribution and can be approximated as, $\operatorname{Normal}\left(N_{1} L_{1}, N_{1} \sigma_{L_{1}}^{2}\right)$. Therefore, in a $99.9 \%$ confidence interval for $N_{1}$ desynchronized flows aggregate preambles required can be approximated by $L_{1} \pm 3.3 \alpha \frac{L_{1}}{\sqrt{N_{1}}}$, where $\alpha$ depends upon $i($.$) and d($.$) . Therefore, we advocate that a$ stable solution corresponds to a desynchronization between data flows. In our simulation, we observe that when data flows are desynchronized then we can get away with very small queues in the devices.

\section{System Dynamics and Stability Conditions}

A generalized system dynamics for the proposed adaptive allocation algorithm with a small DS buffer and a round trip delay of $\tau$ can be analyzed by using the following delay differential model (using (2)):

$$
\begin{aligned}
\frac{d L_{1}(t)}{d t} & =\left(i\left(L_{1}(t)\right) b(t-\tau)\right. \\
& \left.-d\left(L_{1}(t)\right)(1-b(t-\tau))\right) \frac{L_{1}(t-\tau)}{\tau},
\end{aligned}
$$

where the functional forms for $i\left(L_{1}(t)\right)$ and $d\left(L_{1}(t)\right)$ determine the preamble increments and decrements procedures, respectively, at the BS. Recall that $b(\cdot)$ is the function of arrivals and successes shown in (9).

Let $L_{1}^{\star}$ be the equilibrium so that $L_{1}(t)=L_{1}^{\star}+y(t)$, where $y(t)=L_{1}(t)-L_{1}^{\star}$ stands for the deviation variable. The 
changes in in $L_{1}(t)$ and $b(t)$ are about a fixed point equilibrium $\left(L_{1}^{\star}, b^{\star}\right)$ which is the solution to $d L_{1}(t)(t) / d t=d b(t) / d t=$ 0 .

Then, linearizing (10) around equilibrium provides

$$
\frac{d y(t)}{d t}+\beta y(t-\tau)+\alpha y(t)=0,
$$

where

$$
\alpha=-\frac{i\left(L_{1}^{\star}\right)}{\tau} b\left(L_{1}^{\star}\right) \text { and } \beta=\frac{i\left(L_{1}^{\star}\right)}{\tau} \frac{L_{1}^{\star} b^{\prime}\left(L_{1}^{\star}\right)}{b\left(L_{1}^{\star}\right)} .
$$

The stability of system dynamics with the proposed adaptive algorithm characterized by (10) is given by the stability of the fixed point of (11) at $y=0$. In reality, the convergence of (11) is based on the roots of the characteristic equations and can be perceived by observing their solutions. We exploit the first order delay differential equations by using the following result from [34].

The following non-linear equation:

$$
\frac{d y(t)}{d t}+\alpha y(t)+\beta y(t-\tau)=0,
$$

where $\alpha \geq 0, \beta>0, \beta>\alpha$, and $\tau>0$, is stable if and only if $2 \beta \tau<\pi$. For the convergence of the proposed adaptive allocation algorithm ((10)), using the above result from [34] and (11), we get

$$
2 i\left(L_{1}^{\star}\right) \frac{L_{1}^{\star} b^{\prime}\left(L_{1}^{\star}\right)}{b\left(L_{1}^{\star}\right)}<\pi .
$$

Using (9) for a small DS buffer, we obtain a sufficient condition for stability as follows:

$$
q_{\max } \frac{i\left(L_{1}^{\star}\right)}{L_{1}^{\star}}<\frac{\pi}{2} .
$$

We can observe that the convergence condition depends on the rate of change of $b\left(L_{1}^{\star}\right)$. Further, we see in (13) that the stability of system dynamics depends on the buffering dynamics at DS devices under the proposed algorithm. The proposed algorithm for adaptive preamble allocation needs to be designed and implemented cautiously in order to guarantee the convergence conditions (the maximum buffer length, $q_{\max }$, and the increase factor $i\left(L_{1}\right)$ are highly dependent on each other). We have shown the graphical interpretation of (13) in Fig. 5. The area below the curve in Fig. 5 indicates a stable region. Moreover, our another important finding in Fig. 5 is the stability-performance tradeoff, as the desirable region for DS devices is always low standing queues, which is attainable only when the factor $\frac{i\left(L_{1}\right)}{L_{1}}$ is high.

\section{Limit Cycle Oscillation}

The occurrence of limit cycles in non-linear systems is quite common. Depending on the parameters, the cycles could be either stable or unstable. Given a stable equilibrium, the analysis of the emergence and stability of such cycles is plausible by using Hopf bifurcation [34].

A Hopf bifurcation occurs at a point where the convergence of a non-linear system dynamics turnabout to periodic limit cycles. Such bifurcation is often local, where the underlying fixed-points representing the dynamical systems lose their

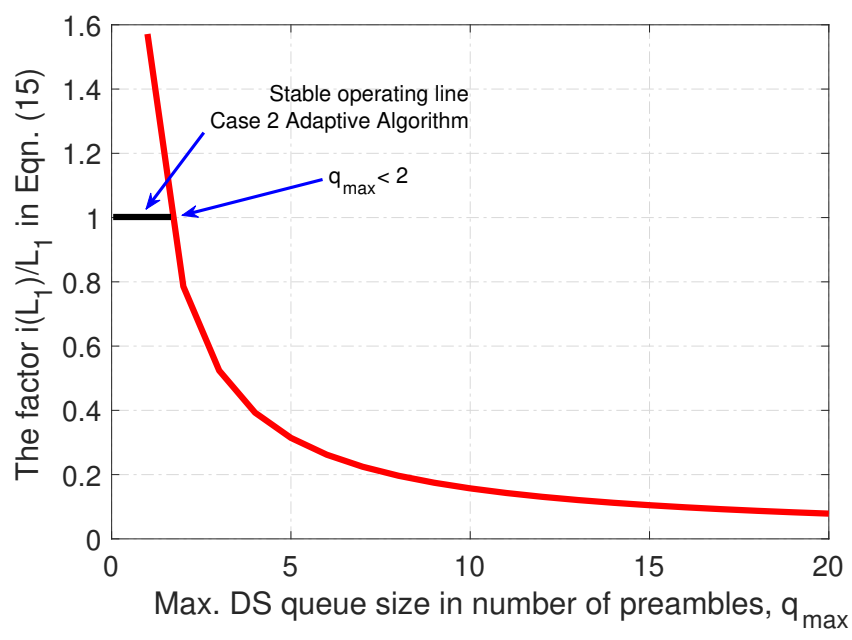

Fig. 5. Graphical interpretation of (13). The area below the curve represents the stable region and that above indicates the unstable region. Observe that the desired region for DS devices is always low standing queues, which are achievable when the factor $i\left(L_{1}\right) / L 1$ is high.

convergence (complex conjugate eigenvalues cross the imaginary axis), and with general hypotheses, a small amplitude limit cycle forks from the fixed-point. In particular, Hopf bifurcation befalls while a periodic solution or limit cycle, encompassing an equilibrium point, arises or moves away (and if one or more parameters change occurs).

For the same non-linear delay differential equation of the system, (12), the system dynamics will show limit cycles as (12) undergoes Hopf bifurcation at

$$
\begin{aligned}
& \tau\left(\beta^{2}-\alpha^{2}\right)^{1 / 2}=\cos ^{-1} \frac{-\alpha}{\beta} \\
& \text { with a period } 2 \pi R T T / \cos ^{-1}(-\alpha / \beta) .
\end{aligned}
$$

Therefore, the condition for Hopf bifurcation associated with the proposed adaptive algorithm and (10) is given by

$$
\frac{i\left(L_{1}^{\star}\right)}{L_{1}^{\star}}\left(q_{\max }^{2}-\left(1-b\left(L_{1}^{\star}\right)\right)^{2}\right)^{\frac{1}{2}}=\cos ^{-1}\left(\frac{1-b\left(L_{1}^{\star}\right)}{q_{\max }}\right) .
$$

\section{E. Adaptive Algorithm Design Guidelines}

In general, an easy way to ensure that stability condition is always met is to mandate the following condition (see Fig. 5):

$$
i\left(L_{1}\right)<\left(\sup _{L_{1}>0} L_{1} \frac{b^{\prime}\left(L_{1}\right)}{b\left(L_{1}\right)}\right)^{-1} \frac{\pi}{2} .
$$

We investigate the following two cases for the choice of system parameters and the adaptive algorithm.

1) Case-1. Additive Increase: With adaptive algorithm parameter

$$
\Delta L_{1}(t)= \begin{cases}i\left(L_{1}(t)\right)=1, & \text { if } \max _{n} q_{n}(t) \geq q_{\max } \\ d\left(L_{1}(t)\right)=1, & \text { o.w. }\end{cases}
$$

a sufficient condition for stability is

$$
\frac{q_{\max }}{L_{1}^{\star}}<\frac{\pi}{2}
$$


and the necessary and sufficient condition is

$$
\frac{1}{L_{1}^{\star}} \sqrt{q_{\max }^{2}-\left(1-b\left(L_{1}^{\star}\right)\right)^{2}}=\cos ^{-1} \frac{\left(b\left(L_{1}^{\star}\right)-1\right)}{q_{\max }} .
$$

2) Case-2. Multiplicative Increase: When the adaptive algorithm evolves as

$$
\Delta L_{1}(t)= \begin{cases}i\left(L_{1}(t)\right)=L_{1}(t), & \text { if } \max _{n} q_{n}(t) \geq q_{\max } \\ d\left(L_{1}(t)\right)=1, & \text { o.w. }\end{cases}
$$

a sufficient condition for the stability is

$$
q_{\max }<\frac{\pi}{2}
$$

and the necessary and sufficient condition is

$$
\sqrt{\left(q_{\max }\right)^{2}-\left(1-b\left(L_{1}^{\star}\right)\right)^{2}}=\cos ^{-1} \frac{b\left(L_{1}^{\star}\right)-1}{q_{\max }} .
$$

Observe in (19) the factor $i\left(L_{1}\right) / L_{1}=1$, therefore the stability does not depend on $L_{1}^{\star}$ (see Fig. 5). For $q_{\max }=1$, the stability of system dynamics with Case 2) is guaranteed (since on an average $q_{\max } \approx 1.5$ is desirable). This is also verified by our graphical representation in Fig. 5, the stable operating line, in this case, is very small and attainable only with smallish $q_{\max }(<2)$. Interestingly, the convergence and divergence of the system dynamics are independent of $L_{1}^{\star}$, which requires further investigations in future work.

\section{F. Practical Applicability of Algorithm 1}

It is worth noting that for deploying Algorithm 1, we need to implement a simple calculation at the BS. In particular, the BS needs to keep track of the number of new arrivals (of access request), $a_{n}(t)$, and compute $p_{n}(t)=\left(1-\frac{1}{L_{1}(t)}\right)^{K_{1}(t)-1}$ to predict $b(t)$. This relation has been quantified mathematically in (9), which identifies the need for tracking $a_{n}(t)$ and $p_{n}(t)$ at the BS. Therefore, $a_{n}(t)$ and $p_{n}(t)$ are available at the BS, and we can observe in (9) that the required $b(t)$ can be estimated without any prior knowledge of $q_{n}(t)$ from the devices.

\section{Simulation Results and Discussion}

We have implemented both Case- 1 and Case- 2 for the proposed adaptive preambles allocation algorithm. After extensive simulations for both the cases under comparable system settings, we have the following findings.

We simulate Case-1 (additive increase) of the adaptive allocation algorithm to analyze the evolution of the system dynamics and preamble allocations when data flows at DS devices are time-varying with the rate $\lambda_{n}(t)$. Fig. 6 demonstrates the results, which shows that the temporal evolution of the max. queue occupancy $\left(\max _{n} q_{n}(t)\right)$ and the variation in the number of preambles allocated for the DS devices are as anticipated. Since the $\max _{n} q_{n}(t)$ is bounded, the access delay is also finite. When $L=50$, we observe that the adaptive algorithm is friendly towards co-existence as more than $50 \%$ preambles would be used for DT devices (considering DT devices are saturated and backlogged with packets) most of

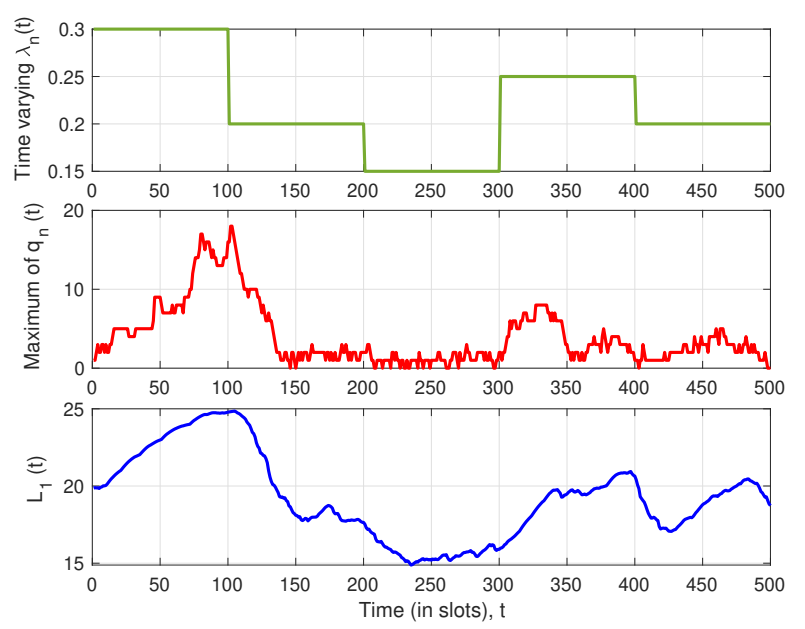

Fig. 6. Evolution of maximum queue occupancy $\left(\max _{n} q_{n}(t)\right)$ compared with that of $L_{1}(t)$ (the number of preambles for DS devices) using Case-1 (additive increase) adaptive algorithm with time-varying $\lambda_{n}(t), \mu=0.01 / N_{1}$ and $N_{1}=30$. Same system settings will be used for Case- 2 in Fig. 7

the times $\left(L_{1}(t)<\frac{L}{2}\right)$; the DS queue occupancy is mostly low standing $\left(\max _{n} q_{n}(t)<10\right.$ packets $)$.

Observe that our design captures the timescales at which the rate of change in queue occupancy and preamble allocations changes over time with the proposed adaptive allocation. Our algorithm balances the result of the change in one being to increase the beneficial effect on the other-the virtuous cycle.

Under the same system settings of Fig. 6, we switch our algorithm to Case 2 (multiplicative increase) to quantify the temporal evolution in queues and the number of preambles. Fig. 7 demonstrates the results, which shows that the temporal evolution of the max. queue occupancy $\left(\max _{n} q_{n}(t)\right)$ and the variation in the number of preambles allocated for the DS devices are very different from that of Case-1 (c.f. Fig. 6). Observe that Case-2 is highly successful in maintaining low standing queues (most of the time $\max _{n} q_{n}(t)<3$ ); however, it requires approximately double the number of preambles. Moreover, the win is, given $L=100$, Case- 2 of the adaptive algorithm is equally friendly towards co-existence as more than $50 \%$ preambles would be used for DT devices most of the time (considering DT devices are saturated and backlogged with packets); the queue occupancy is successfully controlled as desired and guarantees negligible DS queuing delay $\left(\max _{n} q_{n}(t)<3\right.$ packets most of the times). The effects of Case-2 demonstrate that the multiplicative increase is highly responsive (as compared to Case-1) to the temporal changes in the rate of data flows as indicated by the slope of the preamble allocation curves. We can clearly see the difference in the timescales of the changes in $L_{1}(t)$ from Figs. 6 and 7. For tractability and bench-marking, in Fig. 8, we implement the algorithm proposed in [1] and reproduced the result (similar to [1, Fig. 4]), which can be compared directly with Figs. 6 and 7 (the results of our proposed algorithm).

For another set of experiments, we examine the impact of the increase in the number of DS devices given the constant rate of data flows in the devices. Fig. 9 shows that, when 


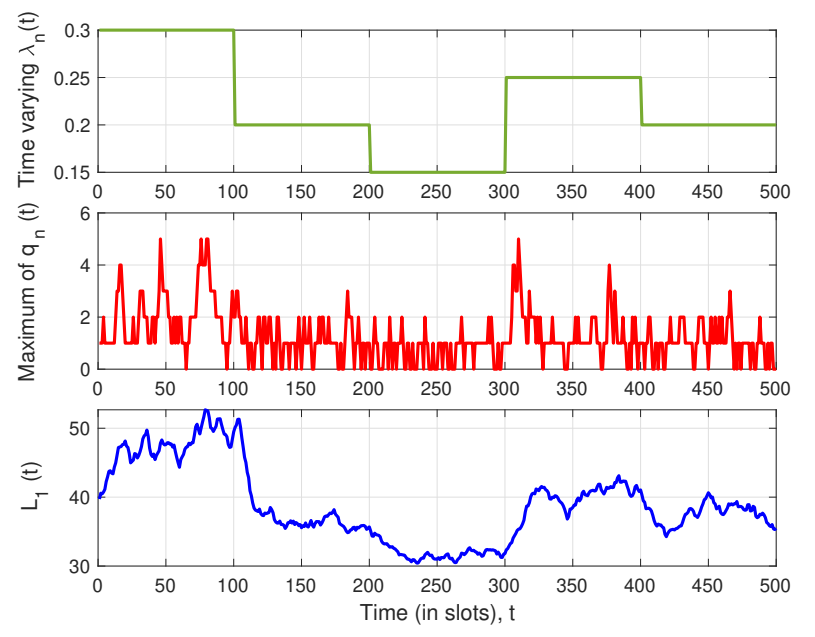

Fig. 7. Evolution of maximum queue occupancy $\left(\max _{n} q_{n}(t)\right)$ compared with that of $L_{1}(t)$ (the number of preambles for DS devices) using Case-2 (multiplicative increase) adaptive algorithm with time-varying $\lambda_{n}(t), \mu=$ $0.01 / N_{1}$ and $N_{1}=30$. Same system settings as that of Fig. 6 .
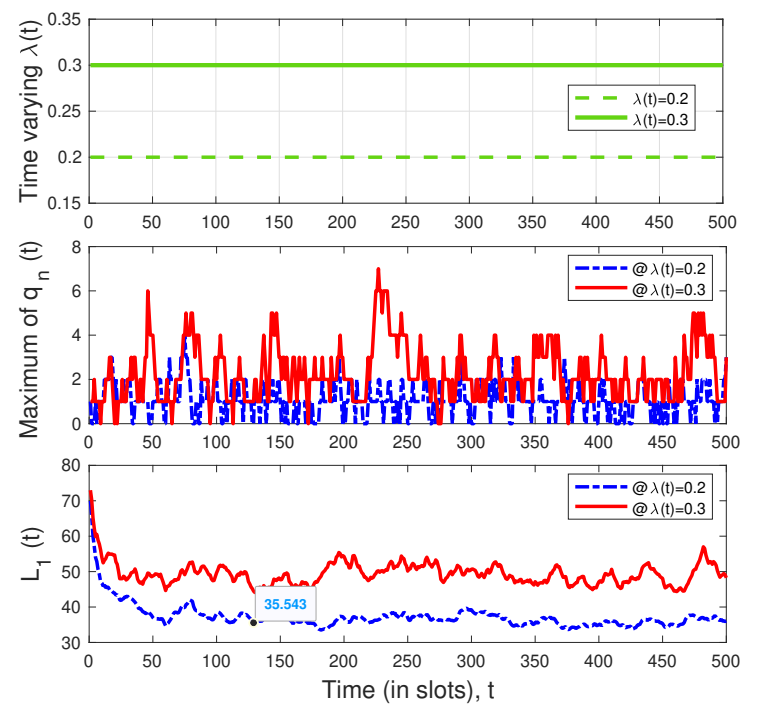

Fig. 8. Bench-marking with [1]: Evolution of maximum queue occupancy $\left(\max _{n} q_{n}(t)\right.$ ) compared with that of $L_{1}(t)$ (the number of preambles for DS devices) using algorithm proposed in [1, Fig. 4] with fixed $\lambda_{n}(t), \mu=$ $0.01 / N_{1}$ and $N_{1}=30$ in two different runs $\left(\lambda_{n}(t)=0.2\right.$ for one run and 0.3 for another run). All other parameter settings are similar as in Figs. 6 and 7.

$\lambda_{n}=0.2$ is fixed, the expected number of preambles allocations initially increases with an increase in the number of DS devices for Case-1 and then remains constant for $\left(N_{1}>80\right)$. Under the same setting, we can see similar behaviour for Case-2 in Fig. 9 - the slope of increase in the expected number of preambles decreases when we switch to Case-2. The corresponding average queue occupancy for both cases are as shown in Fig. 10. It clearly demonstrates that our Case1 adaptive algorithm breaks down when the number of DS devices $N_{1}>65$, however, Case-2 is robust for a large number of devices as well.

For the same setup, when we increase $\lambda_{n}=0.3$, we observe

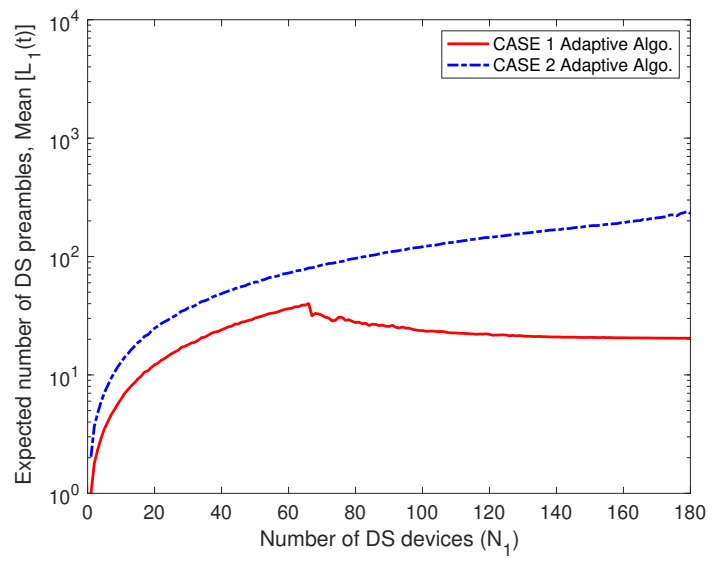

Fig. 9. Comparison of the evolution in the expected number of preambles with an increasing number of DS devices under both cases of the adaptive algorithm when $\lambda_{n}=0.2$.

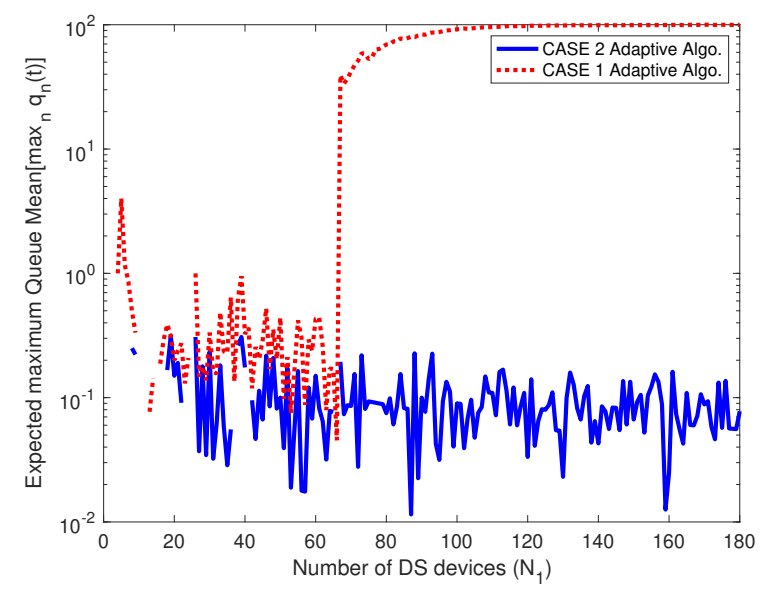

Fig. 10. Comparison of the evolution in the expected number of packets in the queue with an increasing number of DS devices under both cases of the adaptive algorithm when $\lambda_{n}=0.2$. Case- 2 is more robust and scalable in maintaining low queuing delays as compared to Case-1 for a high volume of DS devices as well.

that the effects in Fig. 11 is along the lines of that of Fig. 9. It is worth observing that with Case-2, the mean $L_{1}$ oscillates highly when $N_{1}>160$ in the logarithmic scale, and MATLAB goes out of memory when $N_{1}$ is near 170 . In contrast, with Case-1, even smallish oscillation is observed at $40<N_{1}<60$, it is relatively more scalable. This opens up new avenues for further research and extensive investigations of the other cases (different forms additive and multiplicative schemes and their mix can be analysed other than that of Eqns. (16) and (18)), such as their stability and performance tradeoff.

\section{CONCluding Remarks}

In this paper, we investigated preamble allocation to different types of devices, where fast retrial is adopted for delaysensitive devices so that their access delay can be low, while delay-tolerant machines coexist with them in the system. For stability by the design of the fast retrial approach, we derive stability conditions have using stochastic queuing and controltheoretic ideas. Besides, we developed an adaptive preamble 


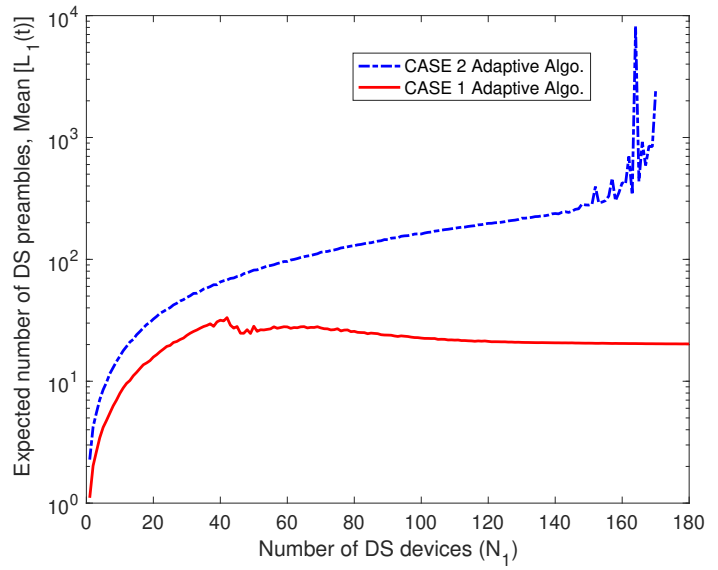

Fig. 11. Comparison of the Evolution in the expected number of preambles with an increasing number of DS devices under both cases of the adaptive algorithm when $\lambda_{n}=0.3$. It is worth observing that with Case-2, the mean $L_{1}$ oscillates highly when $N_{1}>160$ in the logarithmic scale, and MATLAB goes out of memory when $N_{1}$ is near 170 . In contrast, with Case1 , even smallish oscillation is observed at $40<N_{1}<60$, it is relatively more scalable. This opens up new avenues for further research and extensive investigations of the cases which sit between Case-1 and 2 .

allocation algorithm to determine the size of preambles and perform resource pooling dynamically.

We have reported a control-theoretic method to analyze the stability of a system carrying mixed data traffic. The method consists of producing a system of non-linear differential equations, then explaining the system dynamics to understand whether it's stable. A stable solution always corresponds to a desynchronization between data flows. As argued earlier, when data flows are desynchronized then we can get away with minimal queuing in DS devices. We found that the stability depends on queue occupancy, and the actual value of the DS buffer influences the stability of the system dynamics. For example, our Case- 2 rule is too aggressive to ensure high responsiveness, and our Case-1 is sometimes more timid than necessary but ensures smoothness and stability.

Our control-theoretic framework assumes a dynamic system environment, i.e. the time-varying number of devices and data flow configurations that are time-variant. Our adaptive preamble allocation gives a comprehensive measure of success as long as the rate of variability in the system situation is higher than the rate at which our algorithm converges to a solution. The circumstances in which the system setting changes more rapidly calls for new research works.

\section{REFERENCES}

[1] J. Choi, "Fast retrial for low-latency connectivity in MTC with two different types of devices," IEEE Wireless Communications Letters, vol. 9, no. 10, pp. 1786-1789, 2020.

[2] S. Redana et al., "5G PPP architecture working group: View on $5 \mathrm{G}$ architecture," European Commission, 2019.

[3] Sizing up spectrum: using transport the best way with new 5G assets, [online] www.ericsson.com/en/blog/2020/7/sizing-up-spectrumusing-transport-the-best-way-with-new-5g-assets-part2, Ericsson, July 2020.

[4] J. Choi, "On Fast Retrial for Two-Step Random Access in MTC," IEEE Internet of Things Journal, 2020

[5] Study on RAN improvments for machine-type communications, 3GPP TR 37.868 V11.0, October 2011.
[6] Evolved Universal Terrestrial Radio Access (E-UTRA); Medium Access Control (MAC) protocol specification, 3GPP TS 36.321 V13.2.0, June 2016.

[7] C. H. Chang and R. Y. Chang, "Design and analysis of multichannel slotted ALOHA for machine-to-machine communication," in Proc. IEEE GLOBECOM, Dec 2015, pp. 1-6.

[8] J. Choi, "On the adaptive determination of the number of preambles in RACH for MTC," IEEE Communications Letters, vol. 20, no. 7, pp. 1385-1388, July 2016.

[9] H. Jin, W. T. Toor, B. C. Jung, and J. Seo, "Recursive pseudo-Bayesian access class barring for M2M communications in LTE systems," IEEE Trans. Vehicular Technology, vol. 66, no. 9, pp. 8595-8599, 2017.

[10] H. Wu, C. Zhu, R. J. La, X. Liu, and Y. Zhang, "FASA: Accelerated SALOHA using access history for event-driven M2M communications," IEEE/ACM Transactions on Networking, vol. 21, no. 6, pp. 1904-1917, 2013.

[11] S. K. Sharma and X. Wang, "Toward massive machine type communications in ultra-dense cellular IoT networks: Current issues and machine learning-assisted solutions," IEEE Communications Surveys \& Tutorials, vol. 22 , no. 1 , pp. 426-471, 2019.

[12] A. Biral, M. Centenaro, A. Zanella, L. Vangelista, and M. Zorzi, "The challenges of M2M massive access in wireless cellular networks," Digital Communications and Networks, vol. 1, no. 1, pp. 1-19, 2015.

[13] R.-G. Cheng, J. Chen, D.-W. Chen, and C.-H. Wei, "Modeling and analysis of an extended access barring algorithm for machine-type communications in LTE-A networks," IEEE Transactions on Wireless Communications, vol. 14, no. 6, pp. 2956-2968, 2015.

[14] S. Duan, V. Shah-Mansouri, and V. W. Wong, "Dynamic access class barring for M2M communications in LTE networks," in 2013 IEEE Global Communications Conference (GLOBECOM). IEEE, 2013, pp. 4747-4752.

[15] N. Li, C. Cao, and C. Wang, "Dynamic resource allocation and access class barring scheme for delay-sensitive devices in machine to machine (M2M) communications," Sensors, vol. 17, no. 6, 2017. [Online]. Available: https://www.mdpi.com/1424-8220/17/6/1407

[16] S.-Y. Lien, T.-H. Liau, C.-Y. Kao, and K.-C. Chen, "Cooperative access class barring for machine-to-machine communications," IEEE Transactions on Wireless Communications, vol. 11, no. 1, pp. 27-32, 2011.

[17] J.-P. Cheng, C.-h. Lee, and T.-M. Lin, "Prioritized random access with dynamic access barring for RAN overload in 3GPP LTE-A networks," in 2011 IEEE GLOBECOM Workshops (GC Wkshps). IEEE, 2011, pp. 368-372.

[18] T.-M. Lin, C.-H. Lee, J.-P. Cheng, and W.-T. Chen, "PRADA: prioritized random access with dynamic access barring for MTC in 3GPP LTE-A networks," IEEE Transactions on Vehicular Technology, vol. 63, no. 5, pp. 2467-2472, 2014.

[19] M. S. Ali, E. Hossain, and D. I. Kim, "LTE/LTE-A random access for massive machine-type communications in smart cities," IEEE Соттиnications Magazine, vol. 55, no. 1, pp. 76-83, 2017.

[20] C. Zhang, X. Sun, J. Zhang, X. Wang, S. Jin, and H. Zhu, "Throughput optimization with delay guarantee for massive random access of M2M communications in industrial IoT," IEEE Internet of Things J., vol. 6 , no. 6, pp. 10077-10092, 2019.

[21] Q. Du, W. Li, L. Liu, P. Ren, Y. Wang, and L. Sun, "Dynamic RACH partition for massive access of differentiated $\mathrm{m} 2 \mathrm{~m}$ services," Sensors, vol. 16, no. 4, p. 455, 2016.

[22] Y.-J. Choi, S. Park, and S. Bahk, "Multichannel random access in OFDMA wireless networks," IEEE J. Selected Areas in Communications, vol. 24, no. 3, pp. 603-613, March 2006.

[23] J. Choi, "Low-latency multichannel ALOHA with fast retrial for machine-type communications," IEEE Internet of Things J., vol. 6, no. 2, pp. 3175-3185, April 2019.

[24] S. R. Pokhrel, J. Ding, J. Park, O.-S. Park, and J. Choi, "Towards enabling critical mMTC: A review of URLLC within mMTC," IEEE Access, vol. 8, pp. 131 796-131 813, 2020.

[25] Evolved Universal Terrestrial Radio Access (EUTRA) and Evolved Universal Terrestrial Radio Access Network (EUTRAN); Overall Description, TS 36.300 v.14.7.0, 3rd Generation Partnership Project (3GPP), June 2018.

[26] L. Dai, "Stability and delay analysis of buffered ALOHA networks," IEEE transactions on wireless communications, vol. 11, no. 8, pp. 27072719,2012

[27] W. Zhan and L. Dai, "Massive random access of machine-to-machine communications in lte networks: Modeling and throughput optimization," IEEE Transactions on Wireless Communications, vol. 17, no. 4 pp. 2771-2785, 2018. 
[28] S. R. Pokhrel, H. L. Vu, and A. L. Cricenti, "Adaptive admission control for IoT applications in home WiFi networks," IEEE Transactions on Mobile Computing, 2019.

[29] J. Thota and A. Aijaz, "On performance evaluation of random access enhancements for 5G uRLLC," in 2019 IEEE Wireless Communications and Networking Conference (WCNC), 2019, pp. 1-7.

[30] J. Kim, G. Lee, S. Kim, T. Taleb, S. Choi, and S. Bahk, "Two-step random access for $5 \mathrm{G}$ system: Latest trends and challenges," IEEE Network, vol. 35, no. 1, pp. 273-279, 2021.

[31] J. Choi, J. Ding, N.-P. Le, and Z. Ding, "Grant-free random access in machine-type communication: Approaches and challenges," IEEE Wireless Communications, pp. 1-8, 2021.

[32] T. N. Weerasinghe, I. A. M. Balapuwaduge, and F. Y. Li, "Preamble reservation based access for grouped mMTC devices with URLLC requirements," in 2019 IEEE International Conference on Communications (ICC), 2019, pp. 1-6.

[33] S. H. Low, "Analytical methods for network congestion control," Synthesis Lectures on Communication Networks, vol. 10, no. 1, pp. 1-213, 2017.

[34] G. Raina, "Local bifurcation analysis of some dual congestion control algorithms," IEEE Transactions on Automatic Control, vol. 50, no. 8, pp. 1135-1146, 2005.

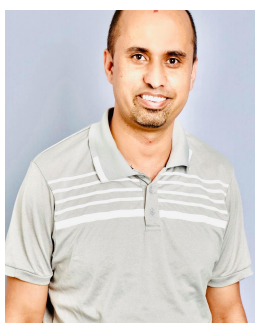

Shiva Raj Pokhrel (M'19) is a Lecturer of Mobile Computing at Deakin University, Geelong, Australia. $\mathrm{He}$ received the B.E./M.E. degree in 2007/2013 from Pokhara University, Nepal and a PhD degree in 2017 from Swinburne University of Technology, Australia. He was a research fellow at the University of Melbourne and a network engineer at Nepal Telecom (2007-2014). His research interests include multi-connectivity, federated learning, industry 4.0 automation, blockchain modelling, optimization, recommender systems, $6 \mathrm{G}$, cloud computing, dynamics control, Internet of Things and cyber-physical systems as well as their applications in smart manufacturing, autonomous vehicles and cities. $\mathrm{He}$ serves/served as the Workshop Chair/Publicity Co-Chair for several IEEE/ACM conferences including IEEE INFOCOM, IEEE GLOBECOM, IEEE ICC, ACM MobiCom, and more. He was a recipient of the prestigious Marie Skłodowska-Curie grant Fellowship in 2017.

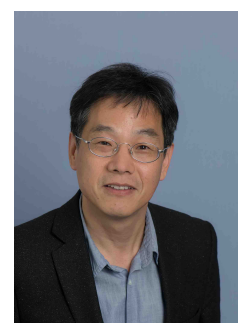

Jinho Choi (SM'02) was born in Seoul, Korea. He received B.E. (magna cum laude) degree in electronics engineering in 1989 from Sogang University, Seoul, and M.S.E. and $\mathrm{PhD}$ degrees in electrical engineering from Korea Advanced Institute of Science and Technology (KAIST) in 1991 and 1994 respectively. $\mathrm{He}$ is with the School of Information Technology, Burwood, Deakin University, Australia, as a Professor. Prior to joining Deakin in 2018, he was with Swansea University, United Kingdom, as a Professor/Chair in Wireless, and Gwangju Institute of Science and Technology (GIST), Korea, as a Professor. His research interests include the Internet of Things (IoT), wireless communications, and statistical signal processing. He authored two books published by Cambridge University Press in 2006 and 2010. Prof. Choi received a number of best paper awards including the 1999 Best Paper Award for Signal Processing from EURASIP. He is on the list of the World's Top 2\% Scientists by Stanford University. Currently, he is an Editor of IEEE Trans. Communications and IEEE Wireless Communications Letters and a Division Editor of Journal of Communications and Networks (JCN). We also had served as an Associate Editor or Editor of other journals including IEEE Communications Letters, JCN, IEEE Trans. Vehicular Technology, and ETRI journal. 